\title{
EMPODERAMIENTO DE LA PERSONA MAYOR MEDIANTE EL EJERCICIO FÍSICO Y LA METODOLOGÍA APRENDIZAJE-SERVICIO (APS)
}

\author{
Pedro Jesús Ruiz-Montero \\ Universidad de Granada \\ pedrorumo@ugr.es \\ Antonio Jesús Casimiro Andújar \\ Universidad de Almería \\ Casimiro@ugr.es
}

Fecha de Recepción: 21 Febrero 2018

Fecha de Admisión: 10 Abril 2018

\section{RESUMEN}

Es necesario abordar un enfoque pedagógico en la educación universitaria, que garantice una calidad mínima de un servicio llevado a cabo con un colectivo social, especialmente las personas mayores. Ello contribuirá a que los beneficios sean máximos tanto en las personas que lo reciben como para quienes lo prestan, en este caso, estudiantes universitarios. El aprendizaje-servicio (ApS) ayuda a entender el contenido del currículo que se pretende impartir a través de contextos reales, potenciando el desarrollo de habilidades sociales-educativas y mejorando la capacidad crítica de los estudiantes. El ejercicio físico como medio posibilitador de experiencias educativas y saludables, ayuda a paliar consecuencias negativas de la edad en el plano psicológico, nutricional, o socioafectivo entre otros. Además, el ApS es un método al que varios autores definen como útil al introducir ejercicio físico como contenido fundamental para propiciar valores como la solidaridad, ayuda, apoyo a la comunidad o cooperación. Por consiguiente, el objetivo del presente trabajo es el de ofrecer una perspectiva diferente de enseñanza. El alumnado universitario aprenderá en un contexto y realidad próximo, a la vez que mejora el plano social, político o físico de la persona mayor. Por consiguiente, estos últimos tendrán una mayor autonomía y control de sus propias decisiones.

Palabras clave: envejecimiento; salud; mejora social; calidad de vida

\section{ABSTRACT} (S-L).

Empowerment of elderly people trough physical exercise and service-learning methodology

It is necessary a pedagogic view in the general and university education. A service focused to a social group should have a minimal quality, specially with elderly people. The service-learning (S-L) 


\section{EMPODERAMIENTO DE LA PERSONA MAYOR MEDIANTE EL EJERCICIO FíSICO Y LA METODOLOGÍA APRENDIZAJE-SERVICIO (APS)}

helps to understand the contents of courses which are teached in real contexts, developing social and educative skills. Physical exercise provides healthy and educative experiences, helping to decrease the negative consequences of bad nutrition, psychologic problems, etc. Furthermore, several authors define the S-L as a useful method to introduce physical exercise as important content if we want to highlight the solidarity, support in a community or cooperation. However, the aim of the present study is offering a different teaching point of view. The students will learn in a real context, as well as there is an improvement in the social, politic or physical factors in the elderly people.

Keywords: ageing; health; social improvement; quality of life

\section{INTRODUCCIÓN}

En los últimos años, todos los trabajos de investigación relacionados con el envejecimiento activo se han centrado en el concepto de capacidad funcional, mejora de la calidad de vida y protagonismo de la propia vida por parte de las personas mayores. La capacidad funcional y autonomía de movimiento de las personas mayores es crucial respecto a la forma en que pueden llevar a cabo las actividades de la vida diaria. La salud y la capacidad funcional son de vital importancia para la calidad de la vida social de las personas: el nivel de capacidad funcional determina la medida en que pueden manejarse con autonomía dentro de una comunidad, participar en distintos eventos, visitar a otras personas, utilizar los servicios que les ofrece la sociedad, y en general, enriquecer sus propias vidas y las de las personas más próximas a ellos.

No hay que olvidar que, en el año 2050, la población global mayor de 60 años rondará los 2.1 billones (Naciones Unidas, 2015). En España, el número de personas mayores de 65 años ha crecido en las últimas décadas considerablemente, pasando de un 13,79\% en 1991 a un 16,61\% en 2007 (Carbonell, Aparicio y Delgado-Fernández, 2009). Por lo tanto, el bienestar general de esta población es importante y el ejercicio físico es un buen aliado, generando experiencias positivas y placenteras, con la mejora de la salud como objetivo principal (Vaquero-Barba, Garay-Ibáñez-DeElejalde y Ruiz-De-Arcaute-Graciano, 2015).

La población mayor y concretamente las mujeres, presentan una característica importante como es la adquisición de hábitos negativos a lo largo de toda la vida (Castillo-Garzón, Ruiz, Ortega y Gutiérrez, 2006). Por consiguiente, este hecho lleva implícito futuras enfermedades y patologías que afectarán negativamente a la edad adulta y mayor de las personas. Entonces, a la hora de planificar y realizar programas de actividades físicas enfocadas a personas mayores, se debe de tener sumo cuidado para respetar siempre las características físicas, fisiológicas y psicosociales (Ruiz-Montero y Baena-Extremera, 2011).

El rol de la mujer ha experimentado cambios profundos en la sociedad, la familia y mundo laboral; también en la práctica física dentro de la sociedad española, originando nuevas formas de relación (Puig y Soler, 2004). La práctica física en el ámbito público mediante diversos programas, ha captado un gran número de mujeres. Sin embargo, siempre se ha de tener en cuenta los recursos y posibilidades económicas de las participantes, ya que siempre no se tiene plena accesibilidad a una práctica de ejercicio físico público (García Ferrando, 2001). Entonces, la práctica de ejercicio físico y adecuadas pautas saludables como alimentación pueden mejorar la calidad de vida de las personas mayores (Ruiz-Montero, Castillo-Rodriguez, Mikalacki, Nebojsa y Korovljev, 2014).

Además, tal y como señala Jiménez (2007), se pone de manifiesto que el nivel de actividad física diaria de los mayores es hoy día insuficiente. Ahora bien, lo importante reside en las motivaciones que tienen las personas mayores para sí hacer actividad física y promover de manera activa hábitos de vida saludable en este colectivo tan heterogéneo y en permanente cambio social y cultural. Esto llevará a una mayor autonomía de la persona y, por consiguiente, empoderamiento de cara 
al día a día. Así pues, para definir el concepto de calidad de vida y autonomía activa en los mayores, se deberían plantear cinco categorías generales como son:

- Bienestar físico: refiriéndose a la comodidad en términos materiales, de higiene, salud y seguridad.

- Relaciones interpersonales: con referencia a las relaciones familiares y el grado de

Integración social del sujeto.

- Desarrollo personal: que incluiría el desarrollo de todas las dimensiones del individuo, esto es, intelectual, autoexpresión, actividad lucrativa y autoconciencia.

- Actividades recreativas: necesarias para mantener la socialización y buscar una recreación pasiva y activa.

- Actividades comunitarias: que incluiría la dimensión de la comunidad en la realización de actividades sociales, educativas y físicas.

Ante estas categorías, lo importante sería plantear que el ejercicio físico puede y debe dar respuesta de manera específica y también de manera transversal a las necesidades que se puedan derivar de la práctica física. En este punto, las personas mayores son agentes sociales activos que requieren de actividades útiles y funcionales, tanto individual como socialmente, por lo que es necesaria la innovación y las perspectivas sistémicas y ecológicas en la acción educativa física como motor positivo de aprendizaje interpersonal y comunitario.

\section{EMPODERAMIENTO Y APRENDIZAJE-SERVICIO (APS)}

Es necesario que la práctica de ejercicio físico esté impregnada por un enfoque pedagógico que garantice una calidad mínima del servicio, ya sea en el contenido de la sesión o/y programa a impartir o por el perfil de los especialistas responsables de dichas sesiones. Además de lo anterior, la práctica de ejercicio físico debe de ser llevada a cabo con un colectivo social para que los beneficios sean máximos tanto en las personas que lo reciben como para quienes lo prestan. Por consiguiente, la aplicación de ApS supone un aprendizaje de valores sociales que ayudan a adaptar estilos de vida de los propios estudiantes a las demandas sociales de su propio contexto y mejorar los aprendizajes personales de las poblaciones receptoras (Gil-Gómez, Chiva-Bartoll y Martí-Puig, 2015).

El rol de un estudiante que participa en un proyecto ApS puede facilitar la evaluación de resultados necesarios para la adaptación del currículo y diversos contenidos en función de la etapa educativa (Alonso Sáez y cols., 2013). También es importante tener en cuenta el clima del grupo-clase, relación establecida entre los estudiantes que llevan a cabo el ApS y estos con el colectivo receptor de ApS (Ruiz-Montero, Chiva-Bartoll y Rivera-García, 2016).

La capacidad física disminuye en las personas mayores y puede venir acompañada de un deterioro psicológico y de las relaciones sociales, por lo que se debe de potenciar un trabajo cooperativo (Lee y Choi, 2015). Esto último justifica la necesidad de trabajar con personas mayores desde todos los ámbitos e instituciones. Los ámbitos de intervención con personas mayores a través de ApS pueden abordar diferentes realidades como la participación ciudadana, patrimonio cultural, solidaridad y cooperación, intercambio intergeneracional o promoción de la salud y calidad de vida. En cuanto la salud y el especial tratamiento de las personas mayores, los estudiantes universitarios pueden responder con soluciones adecuadas mediante una intervención ApS específica a la necesidad y característica de cada grupo de personas mayores.

Cada etapa de la vida tiene su propia y más adecuada pedagogía en la consecución de los objetivos de aprendizaje propuestos, y lo mismo puede ser descrito para el empoderamiento 0 independencia del colectivo mayor para mejorar su salud y calidad de vida a través de una autonomía motriz y mejora capacidad física. Es importante que las personas mayores puedan satisfacer su curiosidad para que puedan adquirir nuevas habilidades físicas, mentales o sociales (Altermann, Martins, 


\section{EMPODERAMIENTO DE LA PERSONA MAYOR MEDIANTE EL EJERCICIO FÍSICO Y LA METODOLOGÍA APRENDIZAJE-SERVICIO (APS)}

Carpes y Mello-Carpes, 2014). Pertenecer a un grupo implica la obligación de socializarse, discutir y defender propias opiniones. Los ambientes de aprendizaje pueden ser aplicados para generar habilidades de trabajo en grupo, colaboraciones, expresar opiniones basadas en principios personales y comunicarse de manera fluida con sus iguales (Escuder-Mollon, 2012); y con ello, un empoderamiento de la persona mayor en el plano social.

Los cambios individuales también tienen cabida en el presente argumento ya que es necesario el conocerse bien a uno mismo en cualquier etapa de la vida. Sin importar la causa, sea biológica, domestica, económica, etc., cualquier cambio debe de ser asimilado, aceptado y cambiado en la medida que sea posible (Chehregosha, 2015). El conocimiento propio y las limitaciones motrices ayudarán a entender mejor el propio cuerpo y capacidad de este a la hora de realizar cualquier tipo de ejercicio físico. Pero uno de los factores más importantes para posibilitar un empoderamiento del mayor es el bienestar físico y la autonomía personal, a sabiendas que el envejecimiento es un proceso irreversible y que normalmente se acompaña de limitaciones físicas entre otros aspectos.

\section{LA PERSONA MAYOR Y EL BENEFICIO DEL APS BASADO EN EJERCICIO FÍSICO}

El American College of Sports Medicine exige que se tengan conocimientos básicos durante el trabajo físico con personas mayores (Nelson y cols., 2007) y para evitar posibles problemas durante el desarrollo de programas de ApS con mayores, la exigencia curricular del alumnado implicado debe de ser alta.

La literatura sobre el beneficio del ApS en voluntarios que ayudan a personas mayores mediante ApS es amplia (Zucchero, 2010), así como la salud percibida en personas mayores receptores de ApS o la reducción de síntomas de depresión en dicho sector (Lum y Lightfoot, 2005). La motivación y autoestima aumenta, considerándose la persona mayor más útil en el contexto que la rodea y estableciendo relaciones sociales más intensas con el alumnado (Ramis, 2014). Si a todo lo anterior se une el condicionante del ejercicio físico, los beneficios en la salud se podrán ver fácilmente. Entonces, no se puede separar la relación del ejercicio físico con la mejora de la salud y la capacidad funcional en personas mayores (Takata et al., 2012), favoreciendo la autonomía física y un empoderamiento a través del movimiento y el ejercicio.

\section{CONCLUSIONES}

La repercusión del ejercicio físico sobre la salud de las personas mayores es considerable, favoreciendo una mayor autoestima, autonomía y motivación personal. Si a este hecho se le suma una metodología ApS llevada a cabo por estudiantes, a través de la práctica de ejercicio físico, el empoderamiento de la población mayor será visible y real, mejorando la situación física, mental, sociocognitiva de la persona mayor

\section{REFERENCIAS}

Alonso Sáez, I. \& cols. (2013) El aprendizaje-servicio en la innovación universitaria. Una experiencia realizada en la formación de educadoras y educadores sociales. Revista Internacional de Educación para la Justicia Social, 2(2), 195-216.

Altermann, C. D. C., Martins, A. S., Carpes, F. P. \& Mello-Carpes, P. B. (2014). Influence of mental practice and movement observation on motor memory, cognitive function and motor performance in the elderly. Brazilian journal of physical therapy, 18(2), 201-209.

Carbonell Baeza, A., Aparicio, V. \& Delgado-Fernández, M. (2009) Involución de la condición física por el envejecimiento. Apunts. Educación Física y Deportes, 162, 98-113.

Castillo-Garzon, M.J., Ruiz, J.R., Ortega, F.B. \& Gutierrez, A. (2006). Anti-aging therapy through fitness enhancement. Clinical Intervention in Aging, 1(3), 213-20. 
Chehregosha, M., Bastaminia, A., Vahidian, F., Mohammadi, A., Aghaeinejad, A., Jamshidi, E. \& Ghasemi, A. (2015). Life Satisfaction Index among Elderly People Residing in Gorgan and Its Correlation with Certain Demographic Factors in 2013. Global Journal of Health Science, 8(8), 41.

Danner, R. \& Edward, D. (1992). Life is movement: Exercise for the older adult. Activities, Adaptation and Aging, 17(2), 15-26.

Escuder-Mollon, P., Esteller-Curto, R., Ochoa, L. \& Bardus, M. (2014). Impact on Senior Learners' Quality of Life through Lifelong Learning. Procedia - Social and Behavioral Sciences, 131, 510516.

García Ferrando, M. (2001). Los españoles y el deporte: prácticas y comportamientos en la última década del siglo XX. Madrid: MECD-CSD.

Gil-Gómez, J., Chiva-Bartoll, 0. \& Martí-Puig, M. (2015) The impact of service learning on the training of pre-service teachers. Analysis from a physical education subject. European Physical Education Review, 21(4), 467-484.

Jiménez, M.C. (2007). Efectos que un programa integral de actividad física dirigida tiene sobre los niveles plasmáticos de lípidos en una población de mujeres de 60 años y más. Jaén: Universidad de Jaén.

Lee, C.-J., \& Choi, Y.-H. (2015). The Effects of a Pilates Exercise Program using Self-Efficacy Sources in Elderly Women. Journal of Environmental Science International, 24(1):117-31.

Lum, T. Y. \& Lightfoot, E. (2005). The Effects of Volunteering on the Physical and Mental Health of Older People, Research on Aging, 27 (11), 31-55.

Naciones Unidas (2015). Envejecimiento Mundial de la población (informe). Asuntos Sociales y Económicos. New York: United Nations.

Nelson, M.E., Rejeski, W.J., Blair, S.N., Duncan, P.W., Judge, J.O., King, A.C., et al. (2007) Physical activity and public health in older adults - Recommendation from the American college of sports medicine and the American heart association. Circulation, 116(9), 1094-1105.

Puig, B.N. \& Soler, P.S. (2004). Mujer y deporte en España: estado de la cuestión y propuesta interpretativa. Apunts Medicina de I Esport, 76, 71-78.

Ramis, A.A. (2014) Personas mayores y aprendizaje-servicio. Barcelona: Zotikos

Ruiz-Montero, P. J. \& Baena-Extremera, A. (2011). Effects of aerobic exercise on the moods in elderly women. Retos. Nuevas tendencias en Educación Física, Deporte y Recreación, 20, 43-47.

Ruiz-Montero, P. J., Castillo-Rodriguez, A., Mikalacki, M., Nebojsa, C. \& Korovljev, D. (2014). 24weeks Pilates-aerobic and educative training to improve body fat mass in elderly Serbian women. Clinical Interventions in Aging, 31(9), 243-248.

Ruiz-Montero, P.J., Chiva-Bartoll, 0. \& Rivera-García, E. (2016). "Aprendizaje-servicio" en los grados de Educación Física: ejercicio físico con personas mayores. Ágora para la Educación Física y el Deporte, 18 (3), 244-258.

Takata, Y, Ansai, T., Soh, I., Awano, S., Yoshitake, Y., Kimura, Y., et al. (2012) Physical fitness and 6.5-year mortality in an 85-year-old community-dwelling population. Arch Gerontol Geriatr, 54(1), 28-33.

Vaquero-Barba, A., Garay-Ibáñez-De-Elejalde, B. \& Ruiz-De-Arcaute-Graciano, J. (2015) La importancia de las experiencias positivas y placenteras en la promoción de la actividad física orientada hacia la salud. Ágora para la educación física y el deporte. 17(2), 168-181.

Zucchero, R.A. (2010). Share Your Experience and I'll Lend You My Ear: Older Adult Outcomes of an Intergenerational Service-Learning Experience, Gerontology and Geriatrics Education, 31(4), 3383-402. 
